\title{
What is the role of affect in the spacing effect?
}

\author{
DAVID G. ELMES, CRAIG J. DYE, and N. J. HERDELIN \\ Washington and Lee University, Lexington, Virginia 24450
}

\begin{abstract}
In Experiments 1 and 2, after studying a list containing connotatively neutral words that were presented once or were presented at various spacing intervals, subjects either attempted free recall or made affective judgments of the study-list targets along a pleasant/unpleasant dimension. Spacing effects occurred in recall, and massed items were judged to be more unpleasant than once-presented and spaced words. In the third experiment, subjects studied homogeneous lists composed of either connotatively good words or connotatively bad ones. Spacing effects were absent in the recall of both types of words because massed-practice words were recalled at a high level, one that was about the same as that for spaced-practice words. Affective judgments were unrelated to presentation condition, and both good and bad massed words were judged to be positive in affect. Although the data suggest that different study conditions can lead to different affective reactions, the results are moot with regard to the relationship between affect and the magnitude of spacing effects.
\end{abstract}

Long-term recall of repeated verbal items increases as the temporal spacing between presentations increases. Theoretical accounts of this pervasive spacing effect (for reviews, see Glenberg, 1979; Hintzman, 1974; Melton, 1970) have focused on various cognitive activities such as encoding, storage, and retrieval. According to these theoretical efforts, processes underlying the spacing effect result from what social psychologists (e.g., Zajonc, 1980) call "cold cognitions." Cold cognitions represent analytic activities and their outcomes, such as feature analysis, rehearsal, and other typical mnemonic activities and internal representations. The general picture derived from research on the spacing effect seems to be that some sort of trace elaboration process and inattention to the second presentation of a massed item are among the most important factors leading to spacing effects (Crowder, 1976; Hintzman, 1976). Exactly why cold cognitions lead to trace multiplexing and failures to attend to massed repetitions has not been well specified. One reason for the failure to determine the causes of the spacing effect may be due to the fact that theories and research have tended to ignore "hot cognitions," which in contrast to cold cognitions include those associated with the affective domain of experience. Boredom, preference, positive arousal, and other affective states and processes have not received much emphasis as factors determining the spacing effect. The present paper reports several experiments designed to test the idea that affective processes play an important role in the spacing effect.

This research was supported by Robert E. Lee research grants. Aspects of these data were discussed at the meetings of the Virginia Academy of Science, Norfolk, 1981, and Blacksburg, 1982. We are grateful for the assistance of $R$. S. Carpenter, P. F. Chapman, and C. F. C. Hall. Direct correspondence to David G. Elmes, Department of Psychology, Washington and Lee University, Lexington, Virginia 24450.
In addition to the possibility mentioned above, a couple of other reasons led us to suspect that affect in addition to ordinary, cold memory processes may underlie the spacing effect. In the first place, a considerable amount of recent research and theorizing has been concerned with the role of affect in a variety of cognitive tasks. For example, Bower (1981), Brown and Kulick (1977), and Zajonc (1980) have detailed the importance of affect in determining performance in several different recall and recognition situations. Perhaps as a reaction to the general cold cognitive approach to memory phenomena, Brown and Kulick as well as Zajonc have argued that affect has a special and, possibly, primary influence on retention. Given these ideas, it seems reasonable to expect that the spacing effect may be, in part, determined by affect.

A more direct reason for suspecting a relationship between affect and spacing effects derives from the results of experiments examining the influence of spacing upon judgments of frequency. Generally, judgments of how frequently a repeated element has occurred are an increasing function of the interpresentation interval (for a review, see Hintzman, 1976). Furthermore, subjective frequency is sometimes less than objective frequency for massed items, and subjective frequency often exceeds objective frequency for spaced events (e.g., Rose, 1980). Since Zajonc and his co-workers (e.g., Zajonc, 1980) have shown that the judged positive affect of a word is directly related to its frequency of experience in the laboratory, it follows that the subjectively more frequent spaced items should be viewed with more positive affect than the subjectively less frequent massed items.

The studies reported below constitute a sequence to test directly the notion that affect is involved in the spacing effect. Suppose the idea that spaced and massed items produce different affective reactions has merit. 
If so, then following presentation of lists containing items with different repetition intervals, subjects allowed to judge those items should indicate that massed ones have lower affect than spaced ones. Thus, the logic of the present research is identical to that of experiments in which frequency judgments are obtained following the presentation of lists containing items with different spacing intervals (e.g., Hintzman, 1976). However, subjects in the present investigation made affective judgments rather than frequency judgments.

In each of the following studies, all subjects heard lists of words containing massed and spaced repetitions. Half the subjects then attempted free recall. The other half made affective judgments of the items, via a modified magnitude-estimation procedure in Experiment 1 and by means of a rating scale in Experiments 2 and 3 . The primary purpose of the first two studies was to determine the affective judgments of massed and spaced words. The third experiment attempted to alter the spacing function by varying the affective connotation of the to-be-remembered words. Subjects heard homogenous lists composed of either very good words or very bad ones. The expectation was that if affect directly controls the spacing effect, then the effect should be changed when affectively "loaded" words are memorized. More specific predictions are detailed subsequently.

\section{EXPERIMENT 1}

\section{Method}

Subjects. Fifty-four undergraduate males at Washington and Lee University volunteered to participate in order to receive extra credit in an introductory psychology course.

Design. Half the subjects heard and then attempted free recall of a study list containing three types of target words: (1) once-presented items, (2) words repeated with a zero lag, and (3) words repeated after seven other list events. The remaining subjects heard the same study list and then judged the pleasantness of the target words by means of a modified magnitude-estimation procedure when the targets were presented again

Materials. A pool of 36 four-letter common nouns was used. These nouns bore no particular semantic relation to each other, and they were judged informally by the experimenters to be neutral in evaluative connotation. Eighteen of the nouns served as targets in the study list, and 12 of them were used as buffers or fillers in that list. The remaining six nouns appeared in the list presented during the judgment task along with the 18 targets from the study list.

List design. In the study list between the five primacy and five recency buffers, there were six targets presented once, six targets presented twice with zero spacing separating each occurrence, and six targets presented twice with seven list events between each presentation. Two fillers were intermixed to maintain the appropriate spacing. The presentation conditions of the targets were equally spaced throughout the list. Three list structures were devised so that each of the three presentation conditons had its second or only presentation just before the first of the recency buffers. The targets were divided into three sets of six words, and across subjects each set appeared equally often in each presentation condition. A total of nine list forms was constructed from the factorial combination of three item sets and three prerecency list structures. These lists were recorded by a male on magnetic tape with the words occurring at a 3-sec rate.

In the judgment list, 24 words were presented once. The nouns included the 18 targets from the study list and the six words that were not presented in that list. One list sequence was recorded by a male on magnetic tape with the words occurring at a 6-sec rate. Order of the words in the judgment list was determined by block randomization, in which each block of four words included a word from each of the presentation condition sets of the study list plus one of the items that did not occur on the study list. Although a given item always occupied the same position during judgment, this confounding assured that the order of judging the study-list conditions was balanced across subjects due to the item-set counterbalancing on the study list.

Procedure. The subjects were tested in groups of three and were assigned to list form and task (free recall or judgment) according to a predetermined random order. Each of the nine forms of the study list was presented to six subjects: Three then attempted free recall, and three performed the judgment task.

Instructions prior to the study list told the subjects to pay close attention to the words because there would be a test at the end. After the final word in the study list, the free recall subjects heard standard free recall instructions and were given unlimited time for written recall.

Immediately following the study list, the judgment subjects received a response sheet and heard magnitude-estimation instructions. For each word they heard in the judgment list, their task was to indicate its subjective magnitude of evaluative connotation by drawing a vertical mark on a 70 -mm horizontal line bracketed by "unpleasant" on the left and "pleasant" on the right. They were told to place their marks according to their opinion concerning the pleasantness or unpleasantness of each word. A mark near "unpleasant" signified that they thought the word was unpleasant, and a mark near "pleasant" indicated that their judgment of that word was a pleasant one. Furthermore, they were instructed to mark the line according to the perceived degree of pleasantness or unpleasantness. The response sheet had 24 lines as described above, 1 line for each of the words in the judgment list. Judgments were subsequently measured to the nearest millimeter from the "unpleasant" end of the horizontal line.

\section{Results}

Free recall. In order to determine the judged affect of massed and spaced items, it is necessary to demonstrate that a spacing effect occurs with the words, list sequences, and instructions that were used. Shown in the bottom panel of Figure 1 are the percentages of target words free recalled. It is apparent that recall varied with presentation condition $[\mathrm{F}(2,52)=16.97$, $p<.001, \eta=.62]$. According to the Newman-Keuls test, recall after a spacing of seven was higher than after zero spacing $(\mathrm{p}<.01)$ and after a single presentation $(p<.01)$. Higher recall occurred after massed repetitions than after a single presentation $(p<.05)$. Thus, the usual advantage of spaced repetitions was found in this experiment.

Judgment. The mean median affective judgments (in millimeters) for the once-presented, massed, spaced, and new words were $33.8,32.2,37$, and 32.6 , respectively. In order to highlight these differences and minimize the differences among the subjects in their willingness to 


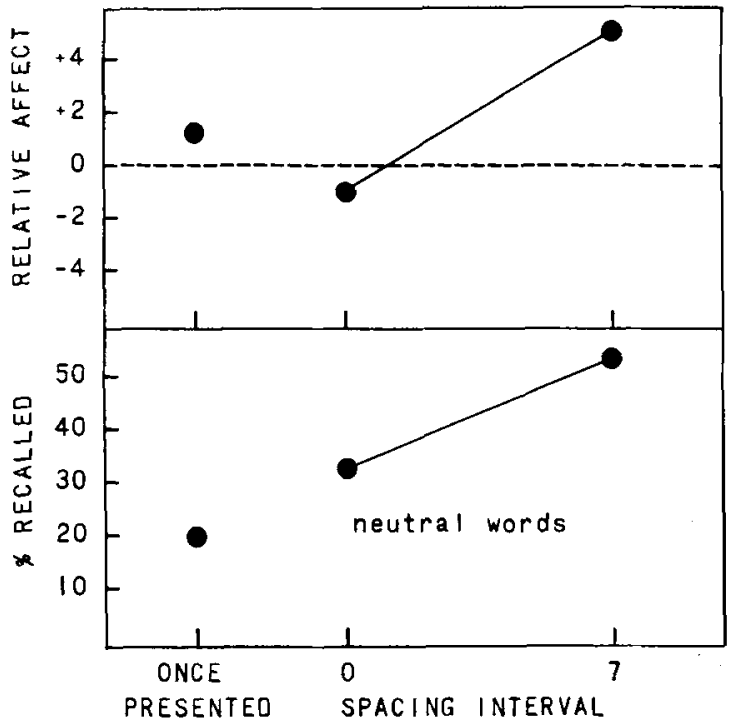

Figure 1. Mean judged relative affect in millimeters (top panel) and mean percentage free recalled (bottom panel) as a function of study-list presentation condition in Experiment 1.

respond "pleasant" or "unpleasant," the judged affect of study-list words was assessed relative to the judged affect of words that did not occur in the study list. Relative affective judgment scores were determined for each subject by subtracting the median judgment of words not presented in the study list from the median judgment of the words from each presentation condition in the study list. Thus, it is possible for a study-list item to have either positive or negative affect relative to words that did not occur on the study list. Means of the difference scores are in the top panel of Figure 1.

Notice that once-presented items and words with a spacing of seven were judged to have positive affect, whereas the massed words were relatively negative in affect. These differences in affect were significant by the Friedman test $\left[\chi^{2} \mathrm{r}(2)=8.91, \mathrm{p}<.01, \mathrm{rm}<.18\right]$. Individual Wilcoxon tests revealed that the spaced items had higher affective ratings than massed ones $[\mathrm{T}(27)=$ $62.7, \mathrm{p}<.01, \mathrm{~m}=.48]$ and items presented once $[\mathrm{T}(27)=98, \mathrm{p}<.05, \mathrm{rm}=.37]$. Massed items received lower affective judgments than did once-presented words $[\mathrm{T}(27)=96, \mathrm{p}<.05, \mathrm{rm}=.37]$.

\section{EXPERIMENT 2}

The second experiment represents a systematic replication of the first. In the second study, different words and spacing intervals occurred in the study list, and a rating scale was used to assess affective judgment. Otherwise, the experimental design, list structure, and procedure were the same as those in Experiment 1.

\section{Method}

Subjects. Forty-four males and 56 females between the ages of 16 and 51 (mean $=23$ ) years were recruited from a local recreation center and paid for their participation.
Materials. The pool of 36 one- and two-syllable nouns came from the Jenkins (1960) norms. The words ranged in evaluative connotation from 3.0 to 3.9 (mean $=3.46$ ), which means they were in the neutral range of the good-bad dimension. Twenty items served as targets and 12 as fillers or buffers in the study list. The remaining four nouns were in the judgment list along with the 20 targets from the study list.

List design. The study list had the same design and counterbalancing features as the ones used in Experiment 1. The major difference was that the current study list had items repeated at spacings of zero, four, six, and eight intervening list events instead of only zero and seven. Four targets were repeated at each of the intervals, and there were four once-presented targets. Twenty-five forms of the study list were determined by the factorial combination of five presentation-condition word sets and five prerecency list structures. These lists were recorded on magnetic tape by a male, with the words occurring at a 3-sec rate.

Construction and recording of the judgment list were the same as in Experiment 1. However, the affective judgment task differed. Instead of a magnitude-estimation task, subjects made affective judgments on a 7-point rating scale bounded by "unpleasant" on the low end and by "pleasant" on the high end.

The subjects were tested in pairs, with two pairs hearing each form of the study list. One pair then engaged in free recall, and the other pair made affective ratings.

\section{Results}

Free recall. The percentages of words free recalled appear in the bottom panel of Figure 2. Recall was higher for repeated items and increased with spacing inter. val $[F(4,196)=11.17, p<.0001, \eta=.43]$. NewmanKeuls tests revealed that all differences between conditions were significant $(p s<.05)$ except for those between spacings of zero and four and between spacings of six and eight.

Judgment. The median affective judgments were $4.5,4.0,4.2,4.2,4.5$, and 4.1 for the once-presented, spacings zero through eight, and new words, respectively. The normalized difference scores relative to the

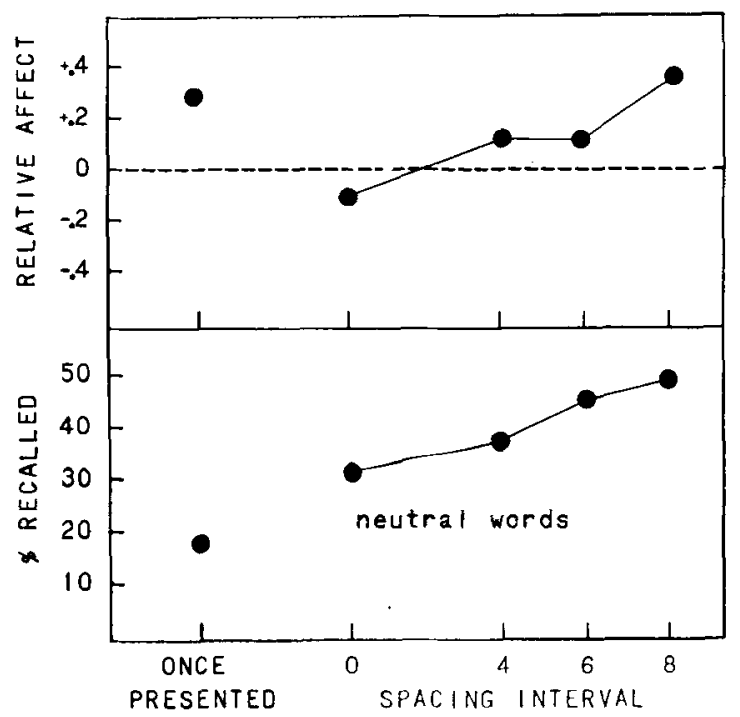

Figure 2. Mean judged relative affect (top panel) and mean percentage free recalled (bottom panel) as a function of studylist presentation condition in Experiment 2. 
words not presented in the study list (the new words) are shown in the upper panel of Figure 2. In terms of general features, these results replicate those found in Experiment 1. Massed items received negative affective ratings and all other words received realtively positive ratings. The overall effects were significant by the Friedman test $\left[\chi^{2} \mathrm{r}(4)=10.16, \mathrm{p}<.05, \mathrm{rm}=.28\right]$. Wilcoxon tests showed that the difference between spacings of zero and eight was significant $[T(50)=403$, $\mathrm{p}<.05, \mathrm{rm}=.28]$, as was the difference between oncepresented words and words with zero spacing $[\mathrm{T}(50)=$ $371.5, \mathrm{p}<.01, \mathrm{rm}=.36]$. No other differences between presentation conditions were statistically reliable.

\section{DISCUSSION OF EXPERIMENTS 1 AND 2}

The pattern of recall results in the two experiments was consistent. Subjects who engaged in free recall following the study list showed the usual advantage of repeated presentation and increased spacing of repetitions. More important, subjects who made affective judgments along a pleasantness/unpleasantness dimension following the study list behaved similarly in the two experiments. Massed-practice items received lower (i.e., more unpleasant) affective judgments than did distributed-practice and once-presented words. When the affective judgments of the study-list words were considered relative to the judgments of words that did not appear in the study list, it was found that massedpractice items were relatively negative in affect, and once-presented and spaced-practiced words were relatively positive in affect. This relationship between affect and spacing was observed both when a magnitudeestimation procedure was used to assess affect (Experiment 1) and when a rating task was used to measure affect (Experiment 2). The congruence of the judgment results from the two procedures attests to the reliability of the effects and also implies that a valid psychometric phenomenon has been measured (e.g., Calfee, 1975).

Granted the reality of the affect/spacing relationship, an important question about these findings immediately arises: Which comes first, changes in affect or changes in recall? It is entirely possible that massed events receive negative judgments because they are remembered poorly. At the time of judgment, subjects might simply give higher, more positive judgments to those words that they remembered best. Differences in affect would thus come about at the time of test rather than at study, and it would mean that the present affect data are mere by-products or epiphenomena of the memory strength resulting from different repetition intervals. A similar sort of argument has been leveled at the finding of different subjective frequencies occurring with differences in spacing. However, the relationship between frequency and spacing seems to arise during study and not when the frequency judgments are made (see,
Hintzman, 1976). We also believe that there is a more fundamental relationship between affect and spacing interval: one that occurs during study and not after the fact.

One reason we believe in such a fundamental relationship comes from results reported by Zajonc and his associates (Zajonc, 1980). They have consistently found that the affective components of a memory trace are more readily and accurately detected than are the cold, cognitive components. Zajonc argues that such results show that affect is a fundamental aspect of the memory trace (cf. Bower, 1981).

A second and more important reason for thinking that differences in recallability are not the primary cause of differences in judged affect comes from a consideration of the affective judgments given to words that received a single presentation in the present studies. In both experiments, once-presented items were judged to be relatively positive in affect, and those items were significantly higher in affect than were massed words. Since items receiving massed repetitions were recalled better than once-presented ones, there must be more to the affect results than can be attributed to a mere by. product of memorability.

Although the dissociation between level of recall and degree of affect may rule out the possibility that differences in affect arise during testing, it does not tell us why there are differences in affect across presentation conditions or whether or not affect directly promotes differences in recall across repetition intervals. We would like to ignore the question of what leads to differences in affect during study and focus on the relationship between affect and level of recall. We do this because a solution to the latter problem is seemingly straightforward. A simpleminded and rather obvious answer to the question of how the degree of affect is related to the level of recall is that people will try to remember events that they like. Put somewhat more formally, affect may alter the mobilization and utilization of cold cognitive resources. What we are suggesting is that affect is a motivational or performance variable much as the magnitude of reward and amount of deprivation are supposed to be performance variables in instrumental conditioning (e.g., Spence, 1956). According to this possibility, the negative affect generated by massed repetitions leads to an unwillingness on the part of the memorizer to engage in the cognitive activities essential for good retention. In contrast, the positive affect that accompanies spaced repetitions results in a more complete employment of mnemonic processes.

The idea that across spacing intervals differences in affect change the motivation to learn and remember is an intriguing one because it suggests a reason for an important component of the spacing effect; namely, subjects do not seem to pay much attention to a massed repetition (Crowder, 1976; Hintzman, 1976). The proposed argument suggests that because of the negative 
affect associated with massing, subjects do not engage in effective processing of the massed repetition. Unfortunately, the results of the first two studies seem to make this hypothesis highly implausible. If affect is supposed to directly influence the level of recall, the higher affect but lower recall of once-presented than massed words certainly clouds the affect/recall contingency. One way out of this dilemna is to assume that affect plays an important role in the spacing effect but not in the advantage associated with multiple presentations. On this view, the advantage in recall due to repeated presentations as compared to a single occurrence results primarily from cold cognitive processes, such as trace elaboration, multiple traces, and the like. In contrast, the advantage in recall due to the spacing of repetitions derives, in part, from the hot cognitive processes outlined above. What we are proposing, therefore, is a two-process mechanism in which cold cognitive factors account for the effectiveness of simply repeating a to-beremembered element, and hot cognitive processes underlie the effectiveness of spacing the repetitions of to-be-remembered elements. Although this is not a very parsimonious theory of repetition effects, portions of the argument are testable and provided the basis for the next experiment.

\section{EXPERIMENT 3}

The third experiment was designed to alter the spacing effect by varying the affective quality of the studylist words. The study list contained either all connotatively good words or all connotatively bad ones. Using such polarized items in the study list should provide an opportunity to observe a variety of interrelationships among affect, spacing, and recall. The good words should be particularly sensitive to the negative affect that accompanies massed practice. If affect directly influences the level of recall, then for the good words we should find a decrease in the recall advantage of massed repetitions over a single presentation. On the other hand, the bad words should be readily shifted in the positive direction by spaced repetition. The enhanced affect should improve recall and led us to expect an increased spacing effect in recall of the bad words.

\section{Method}

Subjects. Seventy-two male undergraduates volunteered to serve in order to receive extra credit in an introductory psychology course. None had participated in either of the first two experiments.

Design. The general design was the same as that used previously: Following a study list, subjects either attempted free recall or made affective judgments. The study list for half the subjects was composed of connotatively good targets, and it was composed of connotatively bad words for the other half. The judgment procedure was the 7-point rating task used in Experiment 2.

List design and Materials. The structure of the study list was the same as in Experiment 1. The words were one- and twosyllable nouns taken from the Jenkins (1960) norms. The good targets had a mean evaluative rating of 5.22 (range $=4.83-5.71$ ), and the bad targets had a mean evaluative rating of 2.11 (range $=$ 1.87-2.47). The new words used in the judgment list had connotative meanings similar to the meaning of the words in their respective study list (for good, mean $=5.21$, range $=4.73-5.56$; for bad, mean $=2.14$, range $=1.91-2.5$ ). Counterbalancing techniques and other aspects of the lists were identical to the previous studies.

Procedure. The subjects were tested in pairs. The assignment of subjects to conditions was by a predetermined random order on the basis of nine list forms (three counterbalancing sets and three prerecency list sequences), task (recall or judgment), and connotative meaning (good or bad study-list items). Other procedural details followed the ones used in the first two studies.

\section{Results and Discussion}

Free recall. Shown in Figure 3 are the recall percentages for the good and bad words. Notice that a spacing effect was not apparent for either type of word, and contrary to expectation, there was a strong repetition effect for both good and bad words. Although the overall effects of presentation condition were significant $[F(2,68)=31.91, p<.0001, \eta=.69]$. NewmanKeuls tests indicated that this was due to higher recall for repeated items than once-presented ones ( $p s<.01$ ). Massed and spaced items did not differ in recall $(p>.1)$. The recall of good and bad words did not differ $(F=$ 1.17); nor was there a significant Presentation Condition by Connotative Meaning interaction $(\mathrm{F}<1)$.

Judgment. The mean median affective judgments of the bad words were $2.5,2.8,2.7$, and 2.6 for the oncepresented, massed, spaced, and new words, respectively. The corresponding values for the good words were $5.4,5.3,5.4$, and 4.9 . The relative affective judgment data were computed as in the first two studies, and they are shown in Figure 4. In contrast to the results of the first two experiments, there does not seem to be any

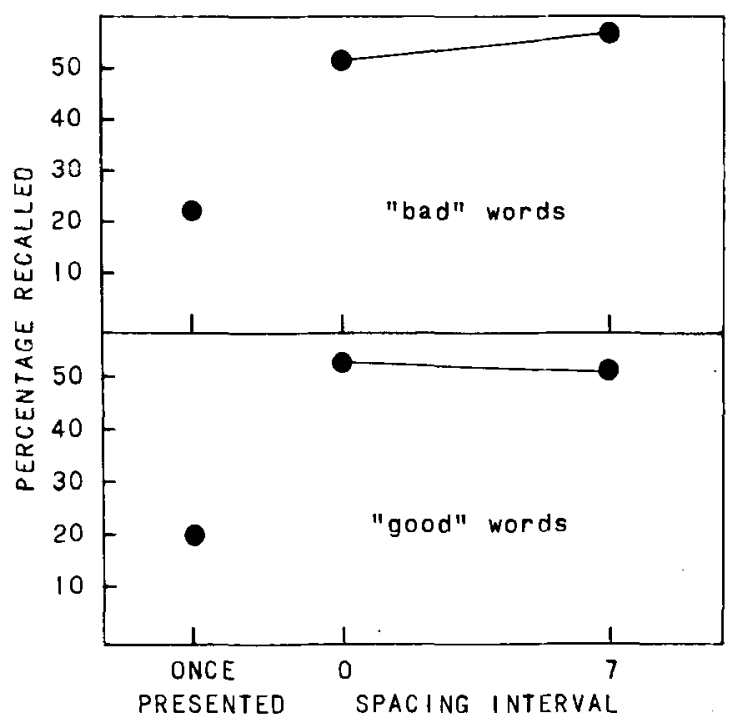

Figure 3. Mean percentages of good and bad words free recalled as a function of study-list presentation condition in Experiment 3. 


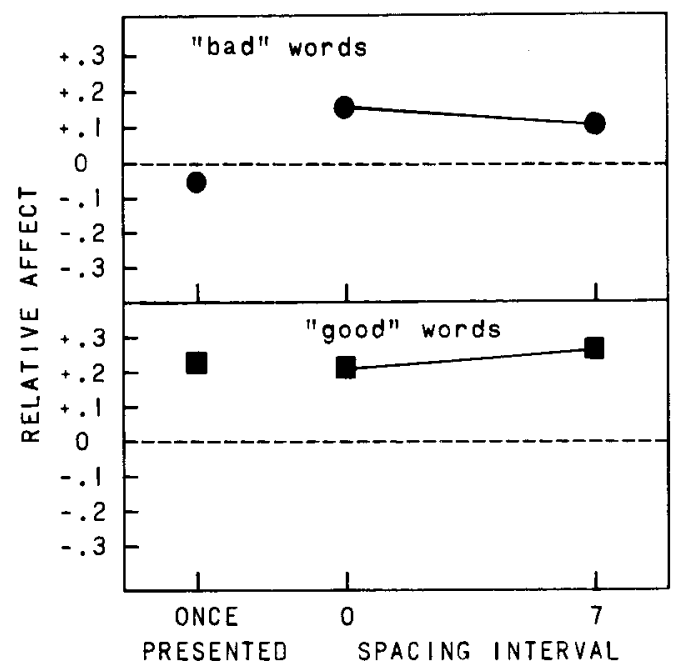

Figure 4. Mean judged relative affect for good and bad words as a function of study-list presentation condition in Experiment 3.

systematic relationship between presentation condition and affective judgment. Separate Wilcoxon tests indicated that affective judgments did not differ among either the good words (ps >.1) or the bad words (ps $>.1$ ).

The predictions were that the recall advantage for massed over once-presented words would be decreased for the good words and that the spacing effect would be enhanced for the bad words. The major results of this experiment are completely contrary to the predictions and are, therefore, puzzling. Instead of a reduction in the repetition effect in the recall of good words, there was an enhancement. Moreover, as shown in Figure 3, massing led to slightly higher recall of the good words than did spaced repetition. Instead of an increase in the spacing effect for bad words, there was a marked attenuation. Massed repetition of the bad words resulted in reliably higher recall than did a single presentation, and the level of recall after massed practice was about the same as that after spaced practice.

There are other aspects of the recall data that are also nonsensical within the framework of the affect-asmotivator hypothesis outlined above. According to that hypothesis, positive affect should enhance recall, which makes it reasonable to expect higher recall of good than of bad words regardless of presentation condition. However, as shown in Figure 3, there was essentially no difference between the percentages of good and bad words that were recalled. Others have also failed to find differential levels of recall for good and bad events (Bower, 1981; Bower, Gilligan, \& Monteiro, 1981). Perhaps a fairer test in the present situation would be a within-list examination of the recall of good and bad words as a function of spacing. At the very least, such a manipulation would provide a contrast between the two types of connotative meaning and should, therefore, enhance any effects that connotative meaning may have on recall.

One reason for the odd results of Experiment 3 may be that affective intensity rather than its qualitative nature is a more important determiner of recall, which makes intuitive sense because we are prone to recall both "highs" and "lows" of our past, apparently on the basis of vividness (Brown \& Kulick, 1977; Neisser, 1982). Furthermore, the affective intensity of an event, and not necessarily its qualitative nature, has been shown to be important in other memory tasks (Bower, 1981; Bower et al., 1981). The words used in Experiment 3 were not selected for their relative degree of affect, but rather for their connotation (good or bad). However, it is possible to get some estimate of their relative intensity of goodness and badness by examining the degree-of-polarization measure $\left(D_{4}\right)$. The greater the degree of polarization, the greater the intensity of connotative meaning. Unfortunately, only a rough estimate of the degree of goodness or badness can be obtained because potency and activity, along with evaluation, determine $D_{4}$ scores (Jenkins, 1960). Nevertheless, given the similar levels of recall for good and bad words, we expect that the degree of polarization of the two sets of words will be very similar if intensity of affect is important. The mean $\mathrm{D}_{4}$ scores were 7.19 and 6.16 for the good and bad words, respectively. The difference between these means is reliable $[t(34)=3.99$, $\left.p=.0006, r_{p b}=.55\right]$, which seems to indicate that the intensity of affect cannot account for the results of this experiment.

Some aspects of the judgment data in Figure 4 are encouraging. In the absence of a spacing effect, there were only minor differences in relative affective judgments across presentation conditions. Therefore, the patterns of judgments observed in the first two experiments probably arose from the spacing manipulation and not during the judgment test. Other aspects of the judgment results are puzzling. As shown in Figure 4, repeated words, regardless of whether they were good or bad, were judged to be pleasant relative to nominally similar words that did not appear in the study list. It is not at all clear why the massed good words are judged positively, especially since they should have been particularly sensitve to the negative affect that is associated with massing. It is also not obvious why massing resulted in higher relative pleasantness for the bad words. Of course, the fact that massed items were recalled well and also received high affective ratings in this experiment fits with the idea that positive affect enhances recall. However, that is a small victory in light of the dramatic refutation of our hypothesis by the remainder of the results.

\section{GENERAL DISCUSSION}

The results of these three experiments are interesting but frustrating. On the one hand, our suspicion that 
affect varied with presentation condition was confirmed. When neutral words are studied, massing leads to negative evaluations and spacing leads to positive judgments (as does a single presentation). On the other hand, however, our attempt to show that affect played a causative role in the spacing effect was a dismal failure. About all we can say about the relationship between affect and recall is that, for repeated items, higher relative affect is associated with higher levels of recall. In the first two experiments, spaced items had higher affect and higher levels of recall than did massed words. In the third study, massed and spaced words were recalled at about the same level, and both had similar degrees of positive affect.

Because of the dissociation between affect and recall across presentation conditions, we are uncertain how to view the relationship between affect and the level of recall. That we are wise to be tentative about the role of affect was confirmed by the results of an experiment in which subjects first free recalled the words and then made affective judgments. The experiment was a replication of Experiment 1, except that the judgment and recall tasks were done by the same subjects and the judgment task was the rating task used in Experiments 2 and 3. If positive affect increases recall, then recalled words should be judged more positively than nonrecalled words. The results showed a spacing effect in recall but similar levels of relative affect for both recalled and nonrecalled words in each presentation condition. Although the data are contaminated by selection effects in a variety of ways, and the judgment data are influenced by the prior recall, we are inclined to accept the results and those of Experiment 3 and conclude that we do not know the role of affect in the spacing effect. At the present time, we do know that there are robust differences in affect resulting from different presentation conditions. The combination of ignorance and knowledge that we now possess is problematic for both theoretical and practical reasons, which we address briefly in turn.

There is no doubt that affective arousal can play an important part in learning and retention (e.g., Rudy, 1974), and, as outlined earlier, the possibility that affect controls processing of repeated elements is a potentially powerful one. Granted these ideas, along with the differential affect across spacing intervals, we are not yet ready to accept the null hypothesis that affect does not enter directly into recall. If it is assumed that affect can influence spacing effects, at least under some conditions, there seem to be two lines of inquiry worth exploring, both of which are based on the supposition that the basic design of the present studies was not appropriate for determining a contingency between affect and recall.

In the first place, it may be that affective judgments should be assessed during study rather than after the completion of study. Such a procedure would require subjects to make affective judgments as the study items were being presented. Although this mode of assessment runs the risk of significantly altering the encoding processes occurring in spacing experiments, a studyphase assessment of affect has the virtue of tying affect to the time of study and would eliminate completely the possibility that affect is a by-product of recall.

A second approach revolves around the well documented mood-congruence effect (Bower, 1981; Bower et al., 1981). This effect is a sort of state-dependent memory based on affect, in which sad events are remembered better when a person is unhappy and pleasant tasks are remembered better when a person is happy. With regard to the current data, the mood-congruence effect suggests that ordinary cognitive processes are more important in determining the spacing effect than is affective arousal because of the emotionally neutral atmosphere in the laboratory. That is, the affective tone of the words generated via spacing is not congruent with the subject's relatively neutral mood. This possibility leads to the interesting prediction of a reversed spacing effect under conditions provoking negative affect in the memorizer.

We believe that the practical implications of our affect/spacing data merit some comment. One of the more distasteful intellectual tasks that can be undertaken by a person is to attempt to master a programmed textbook, especially when the topic is in his or her own area of expertise. The boredom resulting from the easy retrieval of the massed repetitions of small units of information usually generates enough negative affect to terminate reading as soon as possible. Bjork (1975) has already pointed out the inefficiencies associated with the too facile retrieval in programmed materials, and our results indicate that negative affect may be an additional problem. Until the relationships among affect, spacing, and recall are well specified, we will not know how serious a problem exists.

\section{REFERENCES}

Bjonk, R. A. Retrieval as a memory modifier: An interpretation of negative recency and related phenomena. In $\mathbf{R}$. L. Solso (Ed.), Information processing and cognition. Hillsdale, N.J: Erlbaum, 1975.

Bower, G. H. Emotional mood and memory. American Psychologist, 1981, 36, 129-148.

Bower, G. H., Gllligan, S. G., \& Monteiro, K. P. Selectivity of learning caused by affective states. Journal of Experimental Psychology: General, 1981, 110, 451-473.

Brown, R., \& Kulick, J. Flashbulb memories. Cognition, 1977, 5, 73-99.

Calfee, R. C. Human experimental psychology. New York: Holt, Rinehart, \& Winston, 1975.

Crowden, R. G. Principles of learning and memory. Hillsdale, N.J: Erlbaum, 1976.

Glenberg, A. M. Component-levels theory of the effects of spacing of repetitions on recall and recognition. Memory \& Cog. nition, 1979, 7, 95-112.

Hintzman, D. L. Theoretical implications of the spacing effect. In R. L. Solso (Ed.), Theories in cognitive psychology: The Loyola symposium. Potomic, Md: Erlbaum, 1974. 
Hintzman, D. L. Repetition and memory. In G. H. Bower (Ed.), The psychology of learning and motivation (Vol. 10). New York: Academic Press, 1976.

Jenkins, J. J. Degree of polarization and scores on the principal factors for concepts in the semantic atlas study. American Journal of Psychology, 1960, 73, 274-279.

Melton, A. W. The situation with respect to the spacing of repetitions and memory. Journal of Verbal Learning and Verbal Behavior, 1970, 9, 596-606.

Neisser, U. Snapshots or benchmarks? In U. Neisser (Ed.), Memory observed: Remembering in natural contexts. San Francisco: Freeman, 1982.

Rose, R. J. Encoding variability, levels of processing and the effects of spacing of repetitions upon judgments of frequency. Memory \& Cognition, 1980, 8, 84-93.

Rudy, J. W. Stimulus selection in animal conditioning and pairedassociate learning: Variations in the associative process. Journal of Verbal Learning and Verbal Behavior, 1974, 13, 282-296.

Spence, K. W. Behavior theory and conditioning. New Haven: Yale University Press, 1956.

Zajonc, R. B. Feeling and thinking: Preferences need no inferences. American Psychologist, 1980, 35, 151-175.

(Received for publication May 18, 1982; revision accepted October 5, 1982.) 